\title{
Line and continuum variability of two intermediate-redshift, high-luminosity quasars
}

\author{
D. Trevese ${ }^{1}$, D. Paris $^{1}$, G. M. Stirpe ${ }^{2}$, F. Vagnetti ${ }^{3}$, and V. Zitelli ${ }^{2}$ \\ 1 Dipartimento di Fisica, Università di Roma "La Sapienza”, P.le A. Moro 2, 00185 Roma, Italy \\ e-mail: dario.trevese@roma1.infn.it \\ 2 INAF - Osservatorio Astronomico di Bologna, via Ranzani, 40127 Bologna, Italy \\ 3 Dipartimento di Fisica, Università di Roma "Tor Vergata", via della Ricerca Scientifica, 1, 00133 Roma, Italy
}

Received 5 February 2007 / Accepted 30 March 2007

\begin{abstract}
Context. It has been shown that the luminosity of active galactic nuclei and the size of their broad line region obey a simple relation of the type $R_{\mathrm{BLR}}=a L^{\gamma}$, from faint Seyfert nuclei to bright quasars, allowing single-epoch determination of the central black hole mass $M_{\mathrm{BH}}=b L^{\gamma} \Delta_{\mathrm{H}_{\beta}}^{2}$ from their luminosity $L$ and width of $\mathrm{H}_{\beta}$ emission line. Adopting this mass determination for cosmological studies requires the extrapolation to high redshift and luminosity of a relation whose calibration relies so far on reverberation mapping measurements performed for $L \lesssim 10^{46} \mathrm{erg} \mathrm{s}^{-1}$ and redshift $z \lesssim 0.4$.

Aims. We initiated a campaign for the spectrophotometric monitoring of a few luminous, intermediate redshift quasars whose apparent magnitude, $V<15.7$, allows observations with a $1.8 \mathrm{~m}$ telescope, aimed at proving that emission lines vary and respond to continuum variations even for luminosities $\gtrsim 10^{47} \mathrm{erg} \mathrm{s}^{-1}$, and determining eventually their $M_{\mathrm{BH}}$ from reverberation mapping.

Methods. We have repeatedly performed simultaneous spectrophotometric observations of quasars and reference stars to determine relative variability of continuum and emission lines. We describe the observations and methods of analysis.

Results. For the quasars PG 1634+706 and PG 1247+268 we obtain light-curves respectively for CIII] $(\lambda \lambda 1909 \AA), \mathrm{MgII}(\lambda \lambda 2798 \AA)$ and for CIV $(\lambda \lambda 1549 \AA), C I I I](\lambda \lambda 1909 \AA)$ emission lines with the relevant continua. During 3.2 years of observation, in the former case no continuum variability was detected and the evidence for line variability is marginal, while in the latter case both continuum and line variability are detected with high significance and the line variations appear correlated with continuum variations.

Conclusions. The detection of the emission line variability in a quasar with $L \sim 10^{47} \mathrm{erg} \mathrm{s}^{-1}$ encourages the continuation of the monitoring campaign which should provide a black hole mass estimate in another 5-6 years, constraining the mass-luminosity relation in a poorly explored range of luminosity.
\end{abstract}

Key words. galaxies: active - quasars: emission lines - quasars: general - quasar: individual: PG 1634+706 quasars: individual: PG 1247+268

\section{Introduction}

Supermassive black holes (SMBHs) are believed to inhabit most, if not all, the bulges of present-epoch galaxies (Kormendy \& Richstone 1995), and strong evidence exists of a correlation between the black hole mass and either the mass $M_{\text {bulge }}$ and luminosity (Marconi et al. 2003, and refs. therein) or the velocity dispersion $\sigma_{*}$ of the host bulge (Ferrarese \& Merrit 2000; Tremaine et al. 2002). This strongly suggests that the formation and growth of SMBHs and galaxies are physically related processes and provides a basis for a theory of cosmic structure formation, incorporating the feedback from Active Galactic Nuclei (AGNs) (Silk \& Rees 1998; Vittorini et al. 2005, and refs. therein). Black hole mass determinations based on stellar or gas kinematics are intrinsically limited, by angular resolution, to relatively nearby objects. In fact, for bright AGNs the nuclear light prevails over the galactic component, in particular in the central region where the galactic gas or star motion is dominated by the black hole gravitational field. Instead the reverberation-mapping technique is not subjected to this limitation and represents the only means to measure the mass of SMBH in bright AGNs.

Emission lines, in the optical-UV region, are interpreted as a recombination of a gas which is photoionized by the continuum radiation emitted by the inner region of the nucleus, presumably an accretion disk surrounding the black hole. Emission lines respond to variations of the ionizing continuum. Although the physical origin of these variations is poorly known (e.g. Trevese \& Vagnetti 2002; Vanden Berk et al. 2004; De Vries et al. 2005) it is possible to use the response of lines to continuum variations to investigate the structure of the lineemitting region. This requires long campaigns of accurate spectrophotomeric monitoring of AGNs, which have led in the past to major progress towards understanding the physics of the "atmosphere" of Seyfert 1 galaxies. A summary of these results is given in Peterson (1993).

Line widths, e.g. the FWHM $\Delta_{\mathrm{H}_{\beta}}$ of the $\mathrm{H}_{\beta}$ emission line, correspond to rms velocities of the emitting gas clouds. A cross-correlation analysis of continuum and emission-line lightcurves, evidencing a time delay $\tau$ of line with respect to continuum variations, allows us to estimate the size $R=\tau / c$ of the region where the line photons are generated. If the gas motion in the Broad Line Region (BLR) is dominated by gravitation (Peterson \& Wandel 2000), the size estimate $R_{\mathrm{BLR}}$ can be combined with the line width to yield a primary estimate of the virial mass of the black hole $M_{\mathrm{BH}} \propto \Delta_{\mathrm{H}_{\beta}}^{2} / G R_{\mathrm{BLR}}$ and the relevant Eddington ratio. For Seyfert 1 galaxies typical BLR sizes are of the order of light-days to light-weeks. Similar studies are more 
difficult for quasars (QSOs) which require a longer monitoring time.

A long-term campaign for a subsample of 28 QSOs was started in 1991 with the Wise $1.0 \mathrm{~m}$ and the Steward $2.3 \mathrm{~m}$ telescopes (Maoz et al. 1994). As a result, nine years later Kaspi et al. (2000) provided mass estimates for the entire sample. The new data, combined with previous results on Seyfert 1 galaxies, thus spanning a much wider range of intrinsic luminosity, made it possible to establish an average relation between the intrinsic luminosity and the size, $R_{\mathrm{BLR}}=a L^{\gamma}$, with $\gamma \simeq 0.7$, which allows a secondary estimate of the black hole mass based on singleepoch observations. In fact, from luminosity and line width it is possible to derive the black hole mass $M_{\mathrm{BH}}=b L^{\gamma} \Delta_{\mathrm{H}_{\beta}}^{2}$, where both the constant $b$ and $\gamma$ are determined statistically from the available echo-mapping data. Recent studies show that $\gamma$ is in the range $0.5-0.7$, depending on how luminosity is defined, which lines are selected for the echo-mapping, and the fitting procedure adopted (Kaspi et al. 2005; Bentz et al. 2006).

The extreme importance of secondary mass estimates relies on the fact that on the basis of single epoch observations it is possible to study the evolution of the mass distribution of QSOs/AGNs in cosmic time, and to extend the studies of the relation existing between QSOs and host bulges properties. However, the above correlations with primary masses, based on echo-mapping, were established for relatively close and faint AGNs with $z \leq 0.4$ and $\left[\lambda L_{\lambda}(5100 \AA) \lesssim 10^{46} \mathrm{erg} \mathrm{s}^{-1}\right]$, thus it is presently unknown whether they can be extrapolated to higher luminosities and/or redshifts. For instance, the extrapolation of the $M_{\mathrm{BH}}-L$ relation (Kaspi et al. 2000), together with the assumption that the known $M_{\mathrm{BH}}-M_{\text {bulge }}-\sigma_{*}$ relations holds (Tremaine et al. 2002), leads to the prediction of the existence of galaxies with $M_{\text {bulge }} \sim 10^{13.1}-10^{13.4} M_{\odot}$ and $\sigma_{*}$ exceeding $800 \mathrm{~km} \mathrm{~s}^{-1}$. Such galaxies have never been observed, and their existence would put important constraints on galaxy formation models (Netzer 2003). Therefore it is essential to extend the primary mass measures to higher redshifts and luminosities. On the other hand, for high QSO luminosities a large size of the broad line region is expected. This would cause both a smoothing of the line light-curve and larger time delays with respect to continuum variations (Wilhite et al. 2005), thus the very detectability and the amplitude of line variations are open questions.

A sample of objects with redshifts in the range $2<z<3.4$ and apparent magnitude as faint as $m_{V} \sim 18$ is being monitored by Kaspi et al. (2004) with the $9 \mathrm{~m}$ Hobby-Eberly Telescope (HET; Ramsey et al. 1998) and new results were presented recently (Kaspi et al. 2007). During their 6-year monitoring of 6 QSOs, significant continuum and emission-line variations were detected in all targets and a preliminary black hole mass estimation is given for one of them.

In the present paper we describe a new monitoring campaign limited to objects with $V<15.7$ and $1<z<4$ which, thanks to their apparent brightness, can be observed with the mediumsmall 1.82 m Copernicus Telescope at Cima Ekar (Asiago, Italy), through a service mode scheduling of a long term monitoring, and allow investigation of whether: i) echo-mapping is feasible for objects as bright as $\lambda L_{\lambda}(5100 \AA) \sim 10^{47} \mathrm{erg} \mathrm{s}^{-1}$ and ii) the $R_{\mathrm{BLR}}$-luminosity correlation can be extrapolated to such brightness. The paper is organized as follows. Section 2 describes the sample, observations, and the data reduction procedure. Section 3 describes the results for two quasars of the sample. Section 4 summarizes the results and discusses future prospects. In the following we derive $\lambda L_{\lambda}(5100 \AA)$ from the flux in the Johnson $\mathrm{V}$ band, extrapolating the flux density to the
Table 1. The quasars monitored.

\begin{tabular}{lccc}
\hline \hline Object & $z$ & $V$ & $\begin{array}{c}\log \left[\lambda L_{\lambda}(5100 \AA)\right. \\
{\left[\mathrm{erg} \mathrm{s}^{-1}\right]}\end{array}$ \\
\hline APM 08279+5255 & 3.911 & 15.20 & 47.7 \\
PG 1247+268 & 2.042 & 15.60 & 47.0 \\
PG 1634+706 & 1.337 & 15.27 & 46.7 \\
HS 2154+2228 & 1.290 & 15.30 & 46.7 \\
\hline
\end{tabular}

rest-frame $\lambda=5100 \AA$ with a power law $f_{v} \sim v^{-0.5}$, and assuming a standard cosmology $H_{0}=70 \mathrm{~km} \mathrm{~s}^{-1} \mathrm{Mpc}^{-1}, \Omega_{\mathrm{M}}=0.3$, and $\Omega_{\Lambda}=0.7$.

\section{Observations}

\subsection{Object selection}

The sample has been extracted from the Veron-Cetty \& Veron (2003) (11th ed.) catalog with the condition $\delta>0, V<15.7$ mag and $z>1$ in order to select objects of bright enough intrinsic luminosity to investigate the bright end extension of the $R_{\mathrm{BLR}}$ Vs. $\lambda L_{\lambda}(5100 \AA$ ) relation (Kaspi et al. 2000, 2005). These conditions identify 12 objects, only four of which were monitored, owing to the limits on observing time. These four objects are listed in Table 1.

Observations at intermediate redshift make it possible to sample the variability of MgII $\lambda 2798$, CIII] $\lambda 1909$, CIV $\lambda 1559$ lines, instead of $\mathrm{H}_{\alpha}, \mathrm{H}_{\beta}, \mathrm{H}_{\gamma}$ observed in the low redshift study of Kaspi et al. (2000). This allows us to study either BLR at smaller distance from the center, or regions of the same sizes of those producing the Balmer lines, but using lines which respond to different parts of the continuum spectrum. The main emission lines falling within the observed wavelength interval are indicated for two objects in Table 2. Figure 1 shows the average spectra of PG 1634+706 and PG $1247+268$.

With respect to other QSO monitoring programmes, ours is the first which includes the MgII line in part of the observed sources. This line is particularly significant, because i) past Seyfert 1 monitoring campaigns conducted with IUE showed its lag to be similar to that of $\mathrm{H}_{\beta}$ (Clavel et al. 1991; Reichert et al. 1994); ii) this line is most often used to derive estimates of black-hole mass from single-epoch spectra of high- $z$ QSOs (McLure \& Jarvis 2002), because its width is tightly correlated to that of $\mathrm{H}_{\beta}$. Deriving a lag for the $\mathrm{MgII}$ line would therefore allow us to estimate the black-hole mass most consistently with respect to the results of Seyferts, which are mostly based on the monitoring of $\mathrm{H}_{\beta}$.

APM $08279+5255$ is one of the most luminous known QSOs if its emission is considered isotropic. However it has been shown to be lensed by a foreground galaxy (Irwin et al. 1998). Three components, separated by a few tenths of an arcsec have been detected in near-infrared images obtained with Keck telescope and different models of the lensing field predict a few days delay between photometric variations of the components (Ledoux et al. 1998; Ibata et al. 1999; Egami et al. 2000): a short time compared with the expected time scale of intrinsic variations. APM 08279+5255 is also a Broad Absorption Line QSO (Irwin et al. 1998), which makes it more difficult to define regions free from either emission or absorption features to measure continuum variations. The analysis of this object is deferred to a forthcoming paper. HS $2154+2228$ has been observed so far only 5 times and the analysis requires further monitoring. The other two objects PG $1247+268$ and PG1634+706 are 
Table 2. Wavelength intervals for lines and continua $[\AA]$.

\begin{tabular}{lllllllll}
\hline \hline Object & $z$ & Line & $\lambda_{\text {short }, 1}$ & $\lambda_{\text {short }, 2}$ & $\lambda_{1}$ & $\lambda_{2}$ & $\lambda_{\text {long, } 1}$ & $\lambda_{\text {long, } 2}$ \\
\hline PG1634+706 & 1.337 & CIII] $\lambda 1909$ & 4252 & 4298 & 4382 & 4590 & 4630 & 4674 \\
PG1634+706 & 1.337 & MgII $\lambda 2798$ & 6215 & 6285 & 6251 & 6777 & 6755 & 6815 \\
PG1247+268 & 2.042 & CIV $\lambda 1549$ & 4380 & 4502 & 4598 & 4801 & 5202 & 5262 \\
PG1247+268 & 2.042 & CIII] $\lambda 1909$ & 5535 & 5595 & 5770 & 5900 & 6025 & 6085 \\
\hline
\end{tabular}

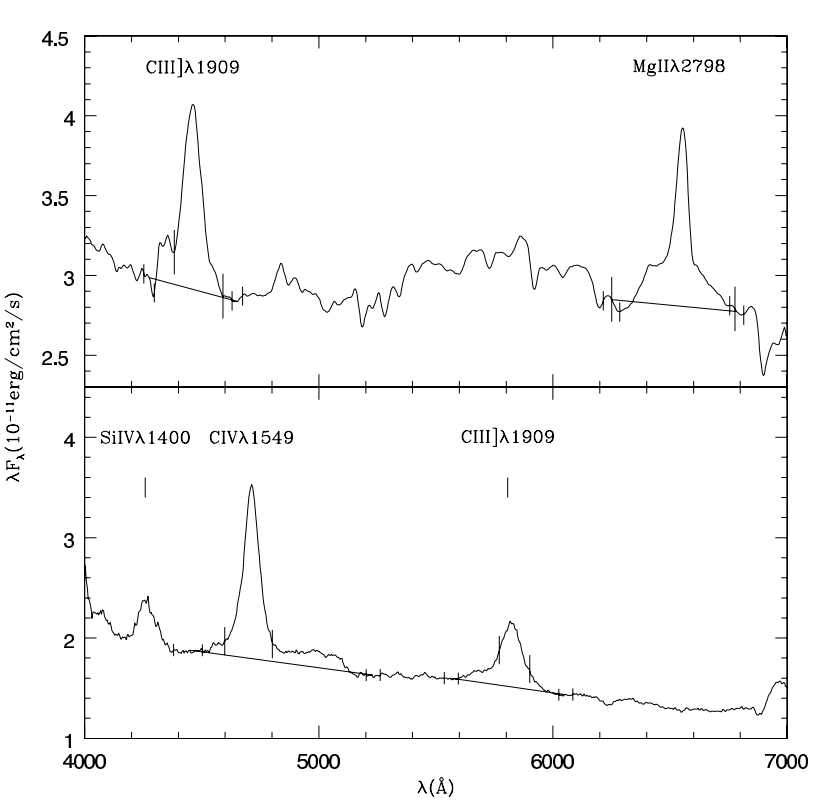

Fig. 1. Average spectra of PG 1634+706 (upper panel) and PG 1247+268 (lower panel). Spectral ranges for continuum determination (short ticks) and line flux evaluation (long ticks), as reported in Table 2, are shown.

analyzed in this paper with the aim of : i) verifying the adequacy of the observational data and reduction procedures, under the assumption that variability amplitudes and characteristic time scales can be extrapolated from the properties of fainter objects; ii) possibly detecting line variations in objects as luminous as $\lambda L_{\lambda}(5100 \AA) \sim 10^{47} \mathrm{erg} \mathrm{s}^{-1}$, and comparing their amplitude with continuum variations. For both objects a star of comparable magnitude, as close as possible to the QSO, has been selected for the relative spectrophotometric calibration which is described in the next section.

\subsection{Spectrophotometric observations and data reduction}

Observations were carried out at Asiago $1.82 \mathrm{~m}$ telescope equipped with the Faint Object Spectrograph \& Camera AFOSC which is a focal reducer with reduction factor of 0.58 , designed to allow quick switching between spectroscopic and imaging modes. The scale at the focal plane is $21.7^{\prime \prime} / \mathrm{mm}$. The detector is a $1024 \times 1024$ thinned CCD array TEK1024 with $22 \times 22 \mu \mathrm{m}^{2}$ pixels corresponding to a scale of $0.473 \operatorname{arcsec}_{\text {pixel }}{ }^{-1}$ and a FOV of $8.14 \times 8.14 \mathrm{arcmin}^{2}$. We adopted a 8.'44-wide slit and a grism with a dispersion of $4.99 \AA$ pixel $^{-1}$, providing a typical resolution of $\sim 15 \AA$ in the spectral range 3500-8450 $\AA$. Spectrophotometric exposures are performed after orienting the slit to include both the QSO and the reference star of comparable magnitude, located at (12:50:11.5 +26:33:32) and (16:34:57.4 $+70: 32: 49)$ (J2000) for PG 1247+268 and PG 1647+706 respectively. The reference stars are included as internal calibrators for
QSO spectra, as described by Maoz et al. (1990) and Netzer et al. (1990). The wide slit is necessary to avoid different fractional losses of the QSO and star light due to possible non perfect slit alignment and differential refraction, which could cause spurious variation of the flux ratios. Lamp flats and $\mathrm{Hg}-\mathrm{Cd}$ ark spectra were also taken for wavelength calibration. At each epoch, typical science observations consist of two consecutive exposures of $1800 \mathrm{~s}$.

QSO and star spectra, $Q(\lambda)$ and $S(\lambda)$ were extracted with the standard IRAF procedures. The QSO/star ratio as a function of wavelength is computed for each exposure $k=1,2$

$\mu^{(k)}(\lambda)=Q^{(k)}(\lambda) / S^{(k)}(\lambda)$.

This quantity is independent of extinction changes during the night. This allows a check of consistency between the two exposures and the rejection of the data if inconsistencies occur (under the assumption that QSO variations are negligible on $1 \mathrm{~h}$ time scale). In fact, typical spectra of two consecutive exposures have a ratio $\mu^{(1)}(\lambda) / \mu^{(2)}(\lambda)$ of order unity, with deviations smaller than 0.02 when averaged over $500 \AA$, at least in the 4000-7000 A range. When discrepancies are larger than 0.04, both exposures are rejected.

It is important to notice that, because of the large slit width, there are small changes in the $\lambda$ scale due to changes of the object position within the slit (which are in general negligible in the case of pairs of consecutive exposures). This led us to proceed as follows. As a first step we register the zero point of the $\lambda$ scale. However, since the changes of position within the slit do not correspond exactly to rigid shifts of the $\lambda$ scale, we select portions of the spectra in wavelength intervals $|\Delta \lambda| \lesssim 1000 \AA$ around each of the QSO emission lines considered (see Table 2), and we determine the shifts for each portion.

Once the wavelength scales are registered, the QSO and star spectra taken in the two exposures are co-added and the ratio

$\mu_{i}(\lambda)=\left(Q_{i}^{(1)}+Q_{i}^{(2)}\right) /\left(S_{i}^{(1)}+S_{i}^{(2)}\right)$

is computed, at each epoch $i$. The reference star is flux calibrated at a reference epoch. Since our aim is to compute relative flux variations, we reduce all quasar spectra to this reference epoch, by multiplying all the $\mu_{i}(\lambda)$ by the flux calibrated star spectrum $f^{\mathrm{S}}(\lambda): f_{i}^{\mathrm{Q}}(\lambda) \equiv \mu_{i}(\lambda) f^{\mathrm{S}}(\lambda)$. We stress that we are not interested in the absolute flux calibration, whose accuracy is of the order of $20 \%$ and which is applied for the sole purpose reporting the QSO spectra in physical units. The star spectrum $f^{\mathrm{S}}(\lambda)$ adopted is the same for all epochs, thus it does not affect the relative flux changes we want to measure.

The QSO spectrum $f^{\mathrm{Q}}(\lambda)=c(\lambda)+l(\lambda)$ can be decomposed in a line $l(\lambda)$ and continuum $c(\lambda)$ spectra. Two values of the continuum, $c_{\text {short }}$ and $c_{\text {long }}$ at shorter and longer wavelengths with respect to the most prominent QSO emission lines, are evaluated in regions as free as possible of other emission features, defined by the wavelengths ranges $\left(\lambda_{\text {short }, 1}, \lambda_{\text {short }, 2}\right)$ and $\left(\lambda_{\text {long, } 1}, \lambda_{\text {long, } 2}\right)$ respectively. Table 2 reports the observer-frame wavelengths 

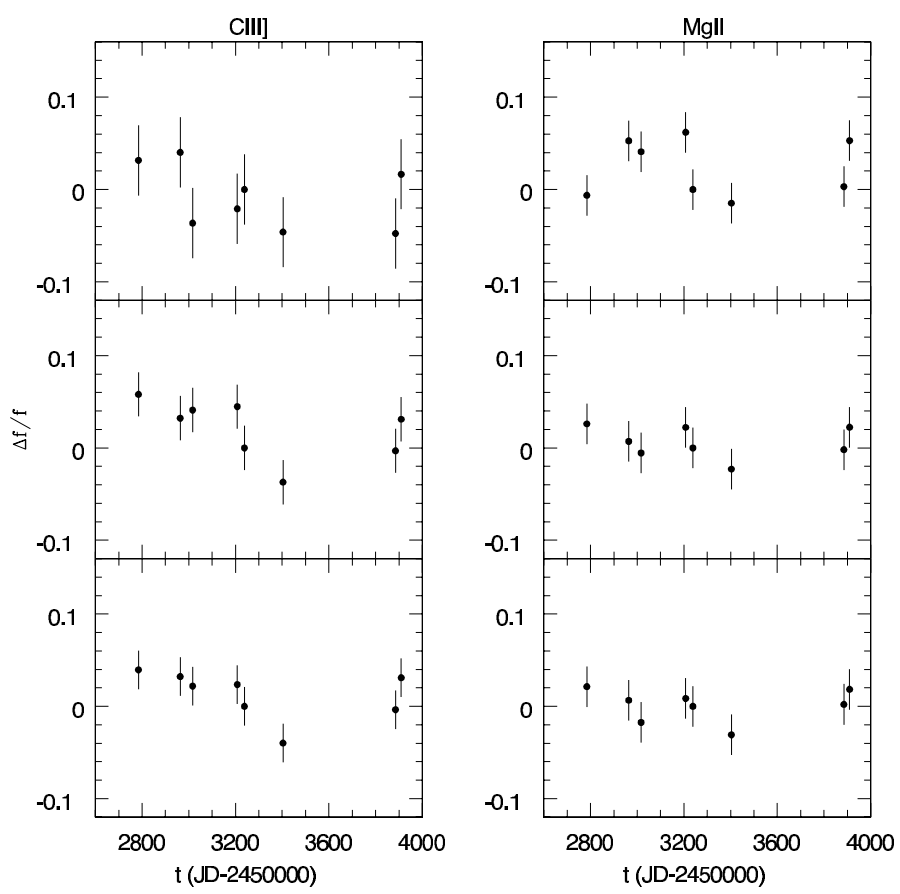

Fig. 2. Light-curves of PG 1634+706 in the observer's frame, as relative flux variations $\Delta f / f$. Upper panels: emission lines, middle panel: shorter wavelength continuum $c_{\text {short }}$, lower panel: longer wavelength continuum $c_{\text {long }}$, left: CIII]/ $\lambda 1909 \AA$, right: MgII $\lambda 2798 \AA$.

defining the continuum regions and the intervals for line integration. These were chosen on the basis of the analysis of UV spectra of low redshift AGNs observed with the International Ultraviolet Explorer (IUE) conducted by Clavel et al. (1991) and by Reichert et al. (1994). Slight modifications were applied to maximize the $\mathrm{S} / \mathrm{N}$ ratio, moreover $c_{\text {long }}$ of $\operatorname{MgII}(\lambda 2798 \AA)$ falls outside the wavelength range covered by the IUE spectrograph.

Line fluxes are computed as:

$f_{l}=\int_{\lambda_{1}}^{\lambda_{2}}\left[F^{(\mathrm{Q})}(\lambda)-c^{\mathrm{int}}(\lambda)\right] \mathrm{d} \lambda$,

where $c^{\text {int }}(\lambda)$ is the linear interpolation through $c_{\text {short }}$ and $c_{\text {long }}$. The extremes $\lambda_{1}$ and $\lambda_{2}$, also listed in Table 2, do not necessarily coincide with $\lambda_{\text {short }} \equiv\left(\lambda_{\text {short }, 1}+\lambda_{\text {short }, 2}\right) / 2$ and $\lambda_{\text {long }} \equiv\left(\lambda_{\text {long }, 1}+\right.$ $\lambda_{\text {long, } 2)} / 2$, and are chosen to optimize the $f_{l}$ signal to noise ratio.

Direct $B$ and $R$ images in $15 \times 15$ arcmin fields centered on the QSOs were taken to check possible variability of the reference star. Comparison of the reference star with the brightest star in the field showed a rms fractional flux variation of 0.02 in both cases. Thus we can assume that the reference star is not variable, at this level of accuracy.

Relative-flux calibration 1- $\sigma$ errors, reported in Figs. 2 and 3, are estimated by adding in quadrature the rms uncertainty on the flux of the reference star to the rms fractional uncertainties of line or continuum fluxes, which, in turn, are computed by comparing pairs of exposures which are added to form quasar spectra of individual epochs.

\section{Results and discussion}

Figures 2 and 3 show the light-curves in the observer's frame, for emission lines and continua listed in Table 2, for PG1634+706 and PG1247+268 respectively. The light curves are expressed in terms of the relative flux variations $\Delta f / f$, with respect to
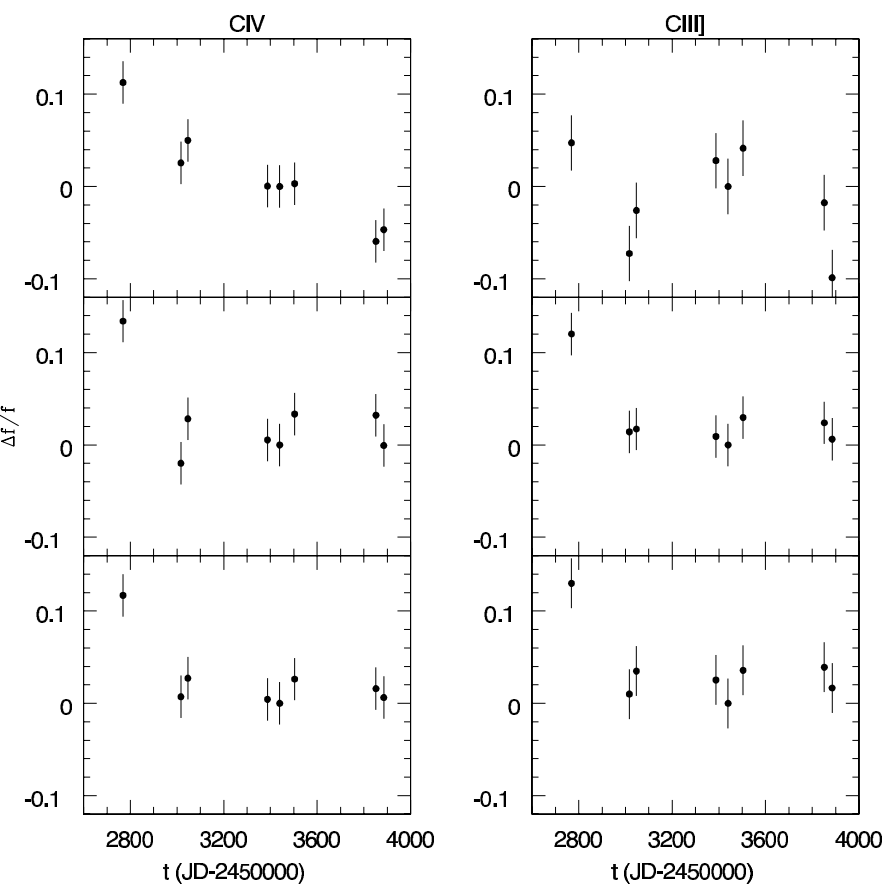

Fig. 3. Light-curves of PG $1247+268$ in the observer's frame, as relative flux variations $\Delta f / f$. Upper panels: emission lines, middle panel: shorter wavelength continuum $c_{\text {short }}$, lower panel: longer wavelength

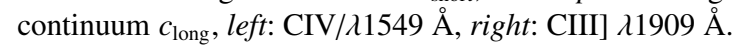

the flux $f$ at the reference epoch where the absolute calibration was performed (see Sect. 2.2). The total time base is 3.3 years in the observer's frame, corresponding in the restframe to $1.4 \mathrm{yr}$ for the former QSOs and $1.1 \mathrm{yr}$ for the latter. In the case of PG1634+706 no significant variations are detected: neither in $\mathrm{CIII}]$ line, which has the lowest $\mathrm{S} / \mathrm{N}$ ratio due to its intrinsic faintness, nor in the MgII line. The relevant continua are also consistent with no variability at a level of $\Delta f / f \sim 0.02-0.03 \mathrm{rms}$.

The result is different in the case of PG $1247+268$. The faintest line CIII] $\lambda 1909 \AA$ shows a marginal evidence of variability and the stronger line CIV $\lambda 1549 \AA$ seems to decrease steadily during the observing period.

An almost steady decrease does not allow us to derive possible line-continuum time-delays from cross-correlation analysis. However we can establish quantitatively the evidence of continuum and, most important, of line variability. For this purpose we define the unbinned discrete structure function

$\operatorname{UDSF}\left(\tau_{i j}\right)=\sqrt{\frac{\pi}{2}}\left|y\left(t_{i}\right)-y\left(t_{j}\right)\right|$

where $y(t)$ represents any of the line or continuum light-curves considered, $t_{i}$ and $t_{j}$ are two observation epochs and $\tau_{i j}=t_{i}-t_{j}$ is the time-delay. The (binned) structure function can be defined in bins of time delay, centered at $\tau$ :

$S(\tau)=\frac{1}{M}\left[\sum_{i, j} U D S F\left(\tau_{i j}\right)\right]$,

where the sum is extended to all the $M$ values of UDSF belonging to a given bin of $\tau$. We adopt the average of UDSF, instead of $\mathrm{UDSF}^{2}$, since it is more stable, i.e. less sensitive to deviant points; the factor $\sqrt{\frac{\pi}{2}}$ in Eq. (4) makes $S(\tau)$ equal to the standard deviation in the case of a Gaussian distribution 


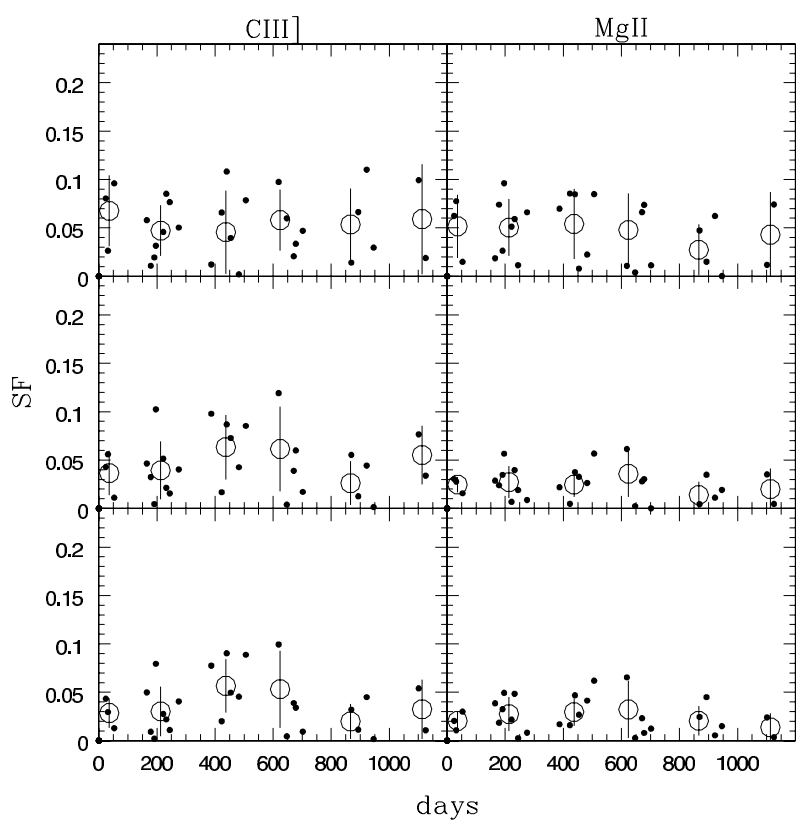

Fig. 4. Structure functions for PG 1634+706 in the observer's frame. Upper panels: emission lines, middle panel: shorter wavelength continuum $c_{\text {short }}$, lower panel: longer wavelength continuum $c_{\text {long }}$, left:

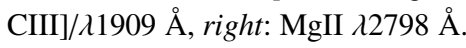

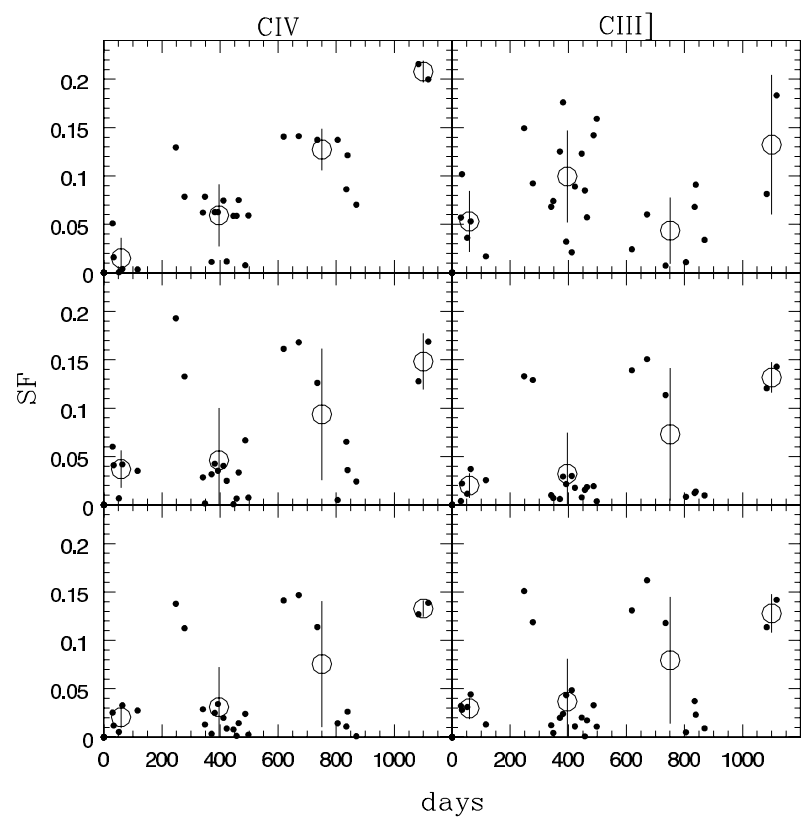

Fig. 5. Structure functions for PG 1247+268. Upper panels: emission lines, middle panel: shorter wavelength continuum $c_{\text {short }}$, lower panel: longer wavelength continuum $c_{\text {long }}$, left: CIV/ $\lambda 1549 \AA$, right: CIII] $\lambda 1909 \AA$.

(see Di Clemente et al. 1996). Structure functions for lines and continua of the two quasars PG 1634+706 and PG 1247+268 are reported in Figs. 4 and 5 respectively, as a function of the time-delay in the observer's frame. The error bars reported in the figures represent simply the standard deviation of the UDSF in each bin and not the uncertainty on their average value. The latter would be $1 /(M-1)$ times the standard deviation, for $M$ uncorrelated UDSF values.

In the case of PG 1634+706, the structure function analysis simply confirms what already appears from the light-curves, namely no significant variations of lines and continua are detected. The rms noise level is about $0.02-0.03$ for continua and 0.05 for lines.

On the contrary, in the case of PG $1247+268$, there is a clear increase of variability for long time lags. To establish the significance of this variability it is necessary to evaluate the probability of the null hypothesis that a value of $S(\tau)$ is produced by pure noise. For this purpose we generated mock noise lightcurves, $n\left(t_{i}\right), i=1, N_{\text {epo }}$, where $N_{\text {epo }}$ is the number of observing epochs, by extracting random numbers with a Gaussian distribution of standard deviation $\sigma$, which represents pure noise, under the assumption that the photometric noise is not correlated on the time scale of our minimum sampling interval (namely about 10 days). Then, the relevant structure function $S_{\text {mock }}(\tau)$ was computed with the same binning adopted for real data. The simulation was iterated for $10^{7}$ times to generate, for each bin, the statistical distribution of the $S_{\text {mock }}(\tau)$ values.

The value adopted for $\sigma$ was estimated, conservatively, from the observed structure functions of Figs. 4 and 5 themselves, as the value of $S(\tau)$ in the first bin. This is, in fact, an overestimate of noise, since it includes short-time-scale variations of the QSO, which however should be negligible on the basis of previous studies of (fainter) QSOs (Giveon et al. 1999; Kaspi et al. 2000). The almost constant value of the structure functions in the case of PG 1634+706 confirms the adopted hypothesis that noise is not correlated on long time scale.

For the CIII] line the rms noise $(\sim 0.05)$ is larger than in the case of the CIV line ( $\sim 0.02)$. This depends on the intrinsic faintness of the CIII] line. According to the above simulations the probability of the null hypothesis, for the second and fourth bins, is $P(>S) \sim 4 \times 10^{-4}$. The local maximum of $S(\tau)$, for CIII] at $\tau \sim 400$ days, is due to an "oscillation" of the light-curve on a time scale comparable with the total time base of the observations. This indicates that a longer time base is needed to properly average individual oscillations and to obtain a "stationary" structure function. Further sampling, trying to avoid yearly periodicity due to periods of optimal observability, is needed to obtain the resolution required for time delay measurements.

The structure function of CIV line shows a much more significant variability: the probability of the null hypothesis in the case of the last two bins, where $S(\tau)$ is 0.13 and 0.21 respectively, is less than $10^{-6}$. The systematic decrease of CIV line luminosity and the relevant continua might seem to rely on the value of the light-curves at the first epoch. Thus we have recomputed the $S(\tau)$ after removing the first epoch. Indeed, in the case of continua the systematic decrease of $S(\tau)$ is no longer evident. On the contrary, for the line it remains practically unchanged (except that the last bin is no longer present). Thus the fact that the probability of the null hypothesis is smaller than $10^{-6}$ does not rely on that particular point in the light-curve.

The main goal of new echo-mapping campaigns is to obtain a direct measure of the central black hole mass and to establish whether the scaling of QSO broad line region size and masses remain the same at higher quasar luminosity. The very detectability of line variation in the most luminous QSOs was, until recently, an open question, since $3 C 273$ with $\left(\lambda L_{\lambda}(5100 \AA) \lesssim\right.$ $\left.10^{46} \mathrm{erg} \mathrm{s}^{-1}\right)$ was the brightest QSO with detected line variability (Kaspi et al. 2005). Evidence of line variability in brighter objects has been reported more recently by Kaspi et al. (2007). The present detection of line variability in one of the two quasars considered strengthens the notion that quasar emission lines respond to continuum changes in high luminosity QSOs like in 
lower luminosity ones and indicates that echo-mapping campaigns are feasible for $\lambda L_{\lambda}(5100 \AA) \gtrsim 10^{47} \mathrm{erg} \mathrm{s}^{-1}$.

Our results are consistent with those of Kaspi et al. (2007), who have monitored sources of comparable luminosities and redshifts. We also detected variability, of comparable amplitude, in the continuum and CIII] and CIV emission lines in one of our sources. Considering the shorter time baseline of our observations, the fact that variability was not found in PG 1634+706 is consistent with the segments without variations which can be seen in the Kaspi et al. (2007) light curves on this time scale.

To estimate the further monitoring needed to measure linecontinuum delays, we should take into account that, on average, the amplitude of variability increases with the rest-frame time lag. However this dependency is weak: $\log S\left(\tau_{\text {rest }}\right) \sim$ $0.15 \log \tau_{\text {rest }}$, according to previous statistical studies on large quasar samples (Vanden Berk et al. 2004; De Vries et al. 2005). In the case of our quasars PG $1634+706$ and PG $1247+268$, the observer's frame $\tau_{\text {obs }}=\tau_{\text {rest }}(1+z)$ is dilated by a factor 2.3 and 3 respectively. The size of the broad line region, based on Balmer line reverberation-mapping, is expected to scale with luminosity as $R_{\mathrm{BLR}} \propto L^{\gamma}$ with $\gamma=0.5-0.7$, depending on how $L$ is measured and the $R_{\mathrm{BLR}}$ vs. $L$ fit is obtained (Kaspi et al. 2000, 2005; Bentz et al. 2006; Kaspi et al. 2007). A Balmer line BLR of about 3 light-years in the rest frame is expected for the relevant luminosities. On the other hand the CIV line corresponds to a BLR size 2 times smaller than Balmer lines (Peterson \& Wandel 2000). Considering that in general a time baseline of about twice the light-crossing time is required in order to obtain a reliable lag from the light curves, we estimate that reverberation mapping should be feasible after 5-6 more years of monitoring.

\section{Summary}

- We initiated a campaign for the monitoring of 4 high luminosity QSOs and we present the results for two of them on the basis of 3.3 years of observation with the $1.82 \mathrm{~m}$ telescope of the Asiago Observatory.

- We discuss the data reduction, the procedures adopted and the level of accuracy attained in relative spectrophotometric variability measurements.

- We perform a structure function analysis of the light-curve and numerical simulations to establish the confidence level of line variability detection.

- We detect line and continuum variability in one of the two QSOs, PG $1247+268$ of $\lambda L_{\lambda}(5100 \AA)=10^{47} \mathrm{erg} \mathrm{s}^{-1}$, with a probability of less than $10^{-6}$ of the null hypothesis that the observed structure function is produced by pure noise.

- This detection supports the notion that emission lines respond to continuum variations as in substantially less luminous QSOs.

The results encourage the continuation of the campaign which should provide time delay and black hole mass estimates in 5-6 years.

Acknowledgements. We acknowledge support from the Asiago Observatory team, in particular Hripsime Navasardian, for observations. We thank the referee for helpful suggestions.

\section{References}

Clavel, J., Reichert, G. A., Alloin, D., et al. 1991, ApJ, 366, 64

Bentz, M. C., Peterson, B. M., Pogge, R. W., Vestergaard, M., \& Onken, C. A. 2006, ApJ, 644,133

De Vries, W. H., Becker, R. H., White, R. L., \& Loomis, C. 2005, ApJ, 129, 615 di Clemente, A., Giallongo, E., Natali, G., Trevese, D., \& Vagnetti, F. 1996, ApJ, 463, 466

Egami, E., Matthews, K., Ressler, M., et al. 2000, ApJ, 535, 561

Ferrarese, L., \& Merrit, D. 2000, ApJ, 539, L9

Giveon, U., Maoz, D., Kaspi, S., Netzer, H., \& Smith, P. S. 1999, MNRAS, 306, 637

Ibata, R. A., Lewis, M. J., Irwin, M. J., Lehar, J., \& Totten, E. J. 1999, ApJ, 118, 1922

Irwin, M. J., Ibata, R. A., Lewis, G. F., \& Totten, E. J. 1998, ApJ, 505, 529

Kaspi, S., Smith, P. S., Meters, H., et al. 2000, ApJ, 533, 631

Kaspi, S., Netzer, H., Maoz, D., et al. 2004, in Coevolution of Black Holes and Galaxies, Carnegie Obs. Astroph. Ser. Vol. 1, ed. L. C. Ho, http://www.ociw.edu/ociw/symposia/series/

Kaspi, S., Maoz, D., Netzer, H., et al. 2005, ApJ, 629, 61

Kaspi, S., Bradt, W. N., Maoz, D., et al. 2007, ApJ, 659, 997

Kormendy, J., \& Richstone, D. 1995, ARA\&A, 33, 581

Ledoux, C., Theodore, B., Petitjean, P., et al. 1998, A\&A, 339, L77

Maoz, D., Netzer, H., Leibowitz, E., et al. 1990, ApJ, 351, 75

Maoz, D., Smith, P. S., Jannuzi, B. T., Kaspi, S., \& Netzer, H. 1994, ApJ, 421, 34

Marconi, A., \& Hunt, L. K. 2003, ApJ, 589L, 21

McLure, R. J., \& Jarvis, M. J. 2002, MNRAS, 337, 109

Netzer, H., Maoz, D., Laor, A., et al. 1990, ApJ, 353, 108

Netzer, H. 2003, ApJ, 583, L5

Peterson, B. M. 1993, PASP, 105, 247

Peterson, B. M., \& Wandel, A. 2000, ApJ, 540, L13

Silk, J., \& Rees, M. J. 1998, A\&A, 331, L1

Ramsey, L. W., et al. 1998, SPIE, 3352, 34

Reichert, G. A., Rodriguez-Pascual, P. M., Alloin, D., et al. 1994, ApJ, 425, 582

Tremaine, S., et al. 2002, ApJ, 574, 740

Trevese, D., \& Vagnetti, F. 2002, ApJ, 564, 624

Vanden Berk, D. 2004, ApJ, 601, 692

Veron-Cetty, M. P., \& Veron, P. 2003, A\&A, 412, 399

Vittorini, V., Shankar, F., \& Cavaliere, A. 2005, MNRAS, 363, 1376

Wilhite, B. C., Vanden Berk, D. E., Kron, R. G., et al. 2005, ApJ, 633, 638 